\title{
DESIGUALDADES SOCIALES EN LA DESNUTRICIÓN CRÓNICA INFANTIL EN EL PERÚ: ANÁLISIS DE RESULTADOS DEL PROGRAMA ARTICULADO NUTRICIONAL 2007-2011
}

\author{
VICTOR AROCENA CANAZAS
}

Resumen.- Este articulo constituye un avance preliminar de una investigación sobre las desigualdades sociales en la salud materno-infantil en el Perú utilizando como fuente de información los resultados del Programa Articulado Nutricional 2007-2011. Se analizan las desigualdades sociales en la desnutrición crónica infantil en el Perú a partir de los indicadores de resultado del Programa Articulado Nutricional 2007-2011. Para tal fin, se utilizan los datos de las Encuestas de Demografia y de Salud Familiar 2007 y 2011 y tres medidas para las desigualdades sociales: cociente entre grupos, diferencia entre grupos e indice de concentración. Los resultados muestran por un lado, una importante disminución en los porcentajes estimados para los indicadores de resultado del Programa Articulado Nutricional entre los años 2007 y 2011. Por otro, la persistencia y, en algunos casos, el aumento en las desigualdades sociales, especialmente en el indicador de resultado final correspondiente a la intervención desnutrición crónica infantil. Se espera que las conclusiones y recomendaciones realizadas en el presente articulo contribuyan al diseño de politicas sobre alimentación y nutrición, principalmente, sobre desnutrición crónica infantil y al diseño del Programa Articulado Nutricional de los próximos años.

Palabras-clave: Desigualdades sociales, desnutrición crónica infantil, programa articulado nutricional, cociente entre grupos, diferencia entre grupos, índice de concentración.

Summary.- This article is a step towards a preliminary research on mother-child social health inequalities in Peru, using as a source of information the results of Nutritional Articulated Program 2007-2011.Social inequalities in child chronic malnutrition in Peru, based on Nutrition Articulated Program 2007-2011 result 
demography and family health 2007 and 2011, and three measures for social inequalities have been used: ratio between groups, the difference between groups and concentration index. On one hand, the results show a significant reduction in the percentages for the outcome indicators of the Nutritional Articulated Program between 2007 and 2011. On the other hand, the persistence and, in some cases, the increase in social inequalities, especially in child related to chronic. malnutrition intervention outcome indicator. It is expected that conclusions and recommendations made in this article may contribute to the design of policies on food and nutrition, primarily on chronic child malnutrition and in the design of Articulated Nutrition Program in the coming years.

Key Words: Social inequalities, child chronic malnutrition, nutritional articulated program, ratio between groups, difference between groups, concentration index.

\section{Introducción}

En las últimas décadas, se ha implementado en nuestro país un conjunto de políticas y se ha llevado a cabo programas -como el Programa Articulado Nutricional- con el propósito de prevenir, atender y mejorar la situación nutricional de la población, principalmente infantil, menor de cinco años. Entretanto, el Informe Perú: Indicadores de Resultados de los Programas Estratégicos 2000-2011, elaborado por el Instituto Nacional de Estadística e Informática (INEI), si bien revela, por un lado, avances significativos en la disminución de los porcentajes de niños con desnutrición crónica, de 22.6 a $15.2 \%$ entre el 2007 y 2011 , por otro lado, revela también, entre otros, la persistencia de importantes desigualdades en la desnutrición crónica según quintiles de bienestar; así, entre el 2007 y 2011 los niños ubicados en el quintil inferior de bienestar fueron afectados de desnutrición crónica en mayor porcentaje( 47.5 y $35.6 \%$ ) comparados con los que pertenecen al quintil superior de bienestar $(4.7$ y $1.8 \%$ ). (INEI, 2012).

La literatura sobre el tema llama la atención sobre las consecuencias de la desnutrición en los niños, las familias y la sociedad. Señalan, entre otros, que la desnutrición infantil durante los primeros años de vida aumenta el riesgo de morbilidad y mortalidad y deteriora el crecimiento y el desarrollo durante la niñez temprana. Los niños afectados ven vulneradas sus capacidades potenciales debido al impacto directo de la desnutrición crónica sobre su 
desarrollo físico y educativo. Su impacto negativo se extiende a la adolescencia y aún a la edad adulta, reduciendo posteriormente su capacidad de trabajo y el rendimiento intelectual, hecho que a su vez puede contribuir a disminuir la productividad económica de las personas, lo que afectará la acumulación del capital humano, el principal capital con que cuenta una sociedad para salir de la pobreza y del sub-desarrollo. (UNICEF, 1998).

La vigencia y permanencia de la desnutrición infantil en nuestro país, principalmente la crónica, independientemente de su cuantía absoluta o relativa, ha constituido y constituye un problema social y de salud pública, problema que el Estado peruano tiene el deber y la obligación de continuar atendiendo. Además del problema social y de salud pública, la desnutrición infantil atenta contra el derecho de nuestros niños a una adecuada alimentación y nutrición, derecho reconocido en diferentes instrumentos legales a nivel internacional y nacional ${ }^{1}$ y constituye una de las expresiones de la inequidad social en el campo de la salud, sobre todo en países en desarrollo como el nuestro, porque es injusta e innecesaria y se presenta, principalmente, en los grupos sociales menos favorecidos. Frente a esta situación, el Estado peruano, por intermedio del Ministerio de Economía y Finanzas (MEF), ha iniciado la implementación del Presupuesto por Resultados (PpR) y su ejecución a través de la puesta en operación de un conjunto de Programas Estratégicos. Así, en 2007 se constituyeron cinco Programas Estratégicos, entre ellos, el Programa Articulado Nutricional (PAN). El PAN comprende un conjunto articulado de intervenciones cuyos resultados organizados en inmediatos e intermedios tienen como resultado final reducir la desnutrición crónica a $16.0 \%$ en el 2011. (MEF, 2008).

Sin embargo, si bien la desnutrición crónica infantil ha disminuido de 22.6 a $15.2 \%$ entre los años 2007 y 2011, alcanzándose el objetivo final programado a nivel nacional, es evidente que aún persisten importantes desigualdades espaciales, sociales y grupales en la desnutrición crónica

A nivel internacional, Declaración Universal de Derechos Humanos (1948); Declaración de los Derechos del Niño (1959); Pacto Internacional de Derechos Económicos, Sociales y Culturales (1966); Convención sobre los Derechos del Niño (1989); Declaración y Plan de Acción sobre la Supervivencia, la Protección y el Desarrollo del Niño ( 1990), Declaración de los Objetivos del Milenio (2000), etc. A nivel nacional, Acuerdo Nacional (2001); Plan Nacional de Superación de la Pobreza (2002); Bases para la Estrategia de Superación de la Pobreza y Oportunidades Económicas para los más Pobres (2003); Estrategia Nacional de Seguridad Alimentaria (2004); Estrategia Nacional de Desarrollo Rural (2004); Programa Nacional de Apoyo Directo a los más Pobres (2005); Estrategia Nacional Crecer (2007), etc. 
infantil. En este contexto, el Programa Estratégico Articulado Nutricional de los próximos cinco años debe, entre otros, rediseñar sus políticas e intervenciones con la finalidad de continuar con la reducción de la desnutrición crónica infantil, sobre todo en las regiones y áreas con elevados porcentajes de desnutrición $\mathrm{y}$, principalmente, reducir las desigualdades sociales que aún persisten.

En este contexto, el presente artículo tiene como principal objetivo estimar la magnitud y analizar las tendencias de las desigualdades sociales existentes en cada una de las intervenciones que comprende el Programa Articulado Nutricional 2007-2011, organizadas según resultados inmediatos, intermedios y final. Se espera que los resultados constituyan, por un lado, un insumo para la toma de decisiones de política social en el área de la alimentación y nutrición y diseño del Programa Articulado Nutricional de los próximos años $\mathrm{y}$, por otro, que contribuya a mejorar las condiciones de salud de la población infantil, sobretodo de aquellos grupos sociales más afectados por la desnutrición crónica infantil.

\section{Aspectos metodológicos}

De acuerdo con la definición de la Organización Panamericana de la Salud (OPS, 1999), desigualdad expresa diferencias sistemáticas, evitables y pertinentes entre los miembros y grupos sociales de una población; entretanto, inequidad significa que la desigualdad observada es además injusta e innecesaria. En este contexto, equidad en la desnutrición crónica infantil en nuestro país implica, entre otros, reducir las desigualdades sociales existentes en la desnutrición crónica infantil, principalmente, en cada una de las intervenciones establecidas en el Programa

Articulado Nutricional 2007-2011. Siguiendo el concepto definido en las Encuestas de Demografia y Salud Familiar (ENDES) se considera como desnutrido crónico a todos aquellos niños menores de cinco años cuya talla para la edad está dos desviaciones estándar por debajo de la media del patrón de crecimiento internacional de referencia del Centro Nacional de Estadísticas Sanitarias de los Estados Unidos (NCHS), establecido como patrón en el Programa Articulado Nutricional 2007-2011.

Las ENDES 2007 y 2011 recolectaron información sobre características de la vivienda, disponibilidad de bienes en el hogar, disponibilidad de ciertos bienes de consumo duradero y servicios directamente relacionados con el nivel socioeconómico. Con esta información, en las ENDES se asigna a cada hogar un puntaje generado mediante la metodología de análisis de componentes principales en función a las caracteristicas de la vivienda y disponibilidad de bienes y servicios. Luego, a los residentes de cada hogar se 
les asigna el valor del hogar en el cual residen. ${ }^{2}$ Esta información ha permitido generar la variable quintiles de bienestar. La propuesta de investigación utilizará la variable quintiles de bienestar para incorporar la dimensión socioeconómica en la estimación de las desigualdades sociales existentes en cada una de las intervenciones del Programa Articulado Nutricional.

El Programa Articulado Nutricional (PAN) elaborado por la Dirección General de Presupuesto Público (DGPP) del Ministerio de Economía y Finanzas (MEF) comprende un conjunto articulado de intervenciones (productos en la terminología de la planificación estratégica) que llevan a resultados inmediatos, intermedios y cuyo resultado final tuvo como objetivo disminuir la desnutrición crónica en niños menores de cinco años de 22.6 en 2007 para $16.0 \%$ en 2011.

\section{Diagrama 1}

\section{Modelo Lógico del Programa Estratégico Articulado Nutricional}

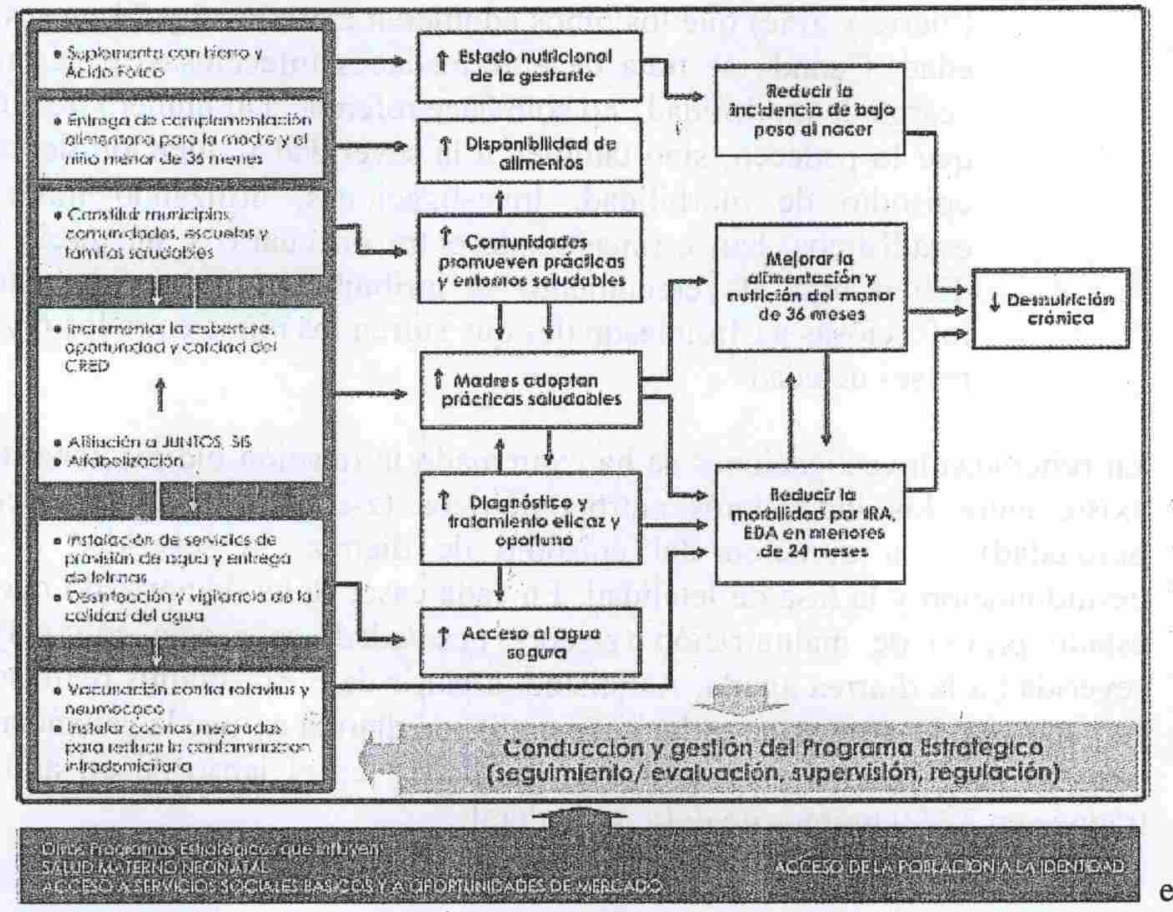

véase Rutstein, Shea O. and Kiersten Johnson. 2004. The DHS Wealth Index. DHS Comparative Reports N ${ }^{\circ} 6$ Calverton, Maryland: ORC Macro; Gwatkin, S. Rutstein, K. Johnson, R. P. Pande y A. Wagstaff. Socio Economic Differences in Health, Nutrition and Population in Perú. The World Bank, mayo 2000. 
En el Diagrama 1, precedente se presenta el Modelo Lógico diseñado para el Programa Articulado Nutricional por el Ministerio de Economía y Finanzas. En el lado izquierdo, se señalan las intervenciones (productos) que el Estado entrega a la población y en el lado derecho, se estructuran los principales resultados a ser obtenidos. La dirección de las flechas, en el recuadro de resultados, revela las relaciones de causalidad que sustenta el diseño de la estrategia del Programa Articulado Nutricional; en cambio, las flechas que vinculan el recuadro izquierdo relacionado con las intervenciones con el recuadro derecho de resultados revelan la teoría de cambio que sustenta el diseño del Programa Articulado Nutricional. (MEF, 2008).

Siguiendo la lógica del Diagrama 1, y por orden de relevancia, la prevalencia de retardo en el crecimiento observado en niños menores de cinco años de edad es el resultado principalmente de:

1. La elevada carga de morbilidad por enfermedades infecciosas (principalmente infección respiratoria aguda y enfermedad diarreica aguda) y por la deficiente ingesta de micronutrientes (hierro y zinc) que los niños adquieren entre los 6 y 24 meses de edad. Cuando se trata de enfermedades infecciosas, el término "carga de morbilidad" no solo hace referencia al número de niños que lo padecen, sino también a la severidad y duración de cada episodio de morbilidad. Investigaciones, utilizando modelos estadísticos, han estimado que entre un cuarto y un tercio del déficit total de crecimiento es atribuible a las enfermedades infecciosas gastrointestinales que sufren los niños entre los 6 y 24 meses de edad. ${ }^{3}$

En reiteradas investigaciones se ha examinado la relación bidireccional que existe entre los indicadores antropométricos (z-score peso/talla, z-score peso/edad) y la duración del episodio de diarrea, la severidad de la deshidratación y la tasa de letalidad. En cada caso, se ha demostrado que el estado previo de malnutrición (z-score peso/edad) se asocia con mayor severidad a la diarrea aguda. Asimismo, a partir de diez estudios realizados en países en desarrollo, se estimó que un día de diarrea reduce la ganancia de peso en 10.9 gramos y que 30 días de diarrea en el lapso de un año se traduce en 327.0 gramos de déficit ponderal.

${ }^{3}$ Martorell, R., Habicht, J.P., Yarbrough, C., Lechtig, A., Klein, R. E. \& Western, K. A. (1975) Acute morbidity and physical growth in rural Guatemala children. Am. J. Dis. Child. 129:1296-1301. 
La deficiencia de micronutrientes es causa y efecto a la vez de la elevada carga de morbilidad. Los sucesivos episodios de diarrea o de infecciones respiratorias agudas ocasionan perdidas de micronutrientes, las cuales no son compensadas por la alimentación habitual que recibe el niño lo que genera la deficiencia de dos minerales fundamentales que son el hierro y el zinc, cuya ausencia retarda el crecimiento de los niños. De episodio a episodio, se va instalando un ciclo pernicioso en el cual la deficiencia de los micronutrientes incrementa la susceptibilidad para adquirir infecciones y ser más resistentes al tratamiento; estas infecciones a su vez causan mayor pérdida de estos micronutrientes. En la actualidad, está ampliamente documentada la relación de causalidad entre deficiencia de zinc con la duración y severidad de la diarrea. (MEF, 2008).

2. La inadecuada alimentación del niño en los primeros 6 meses de vida en base a la lactancia materna exclusiva, seguida de la inadecuada introducción de alimentos en cuanto a la frecuencia, la composición, la cantidad, la preparación y la manera de alimentar al menor de edad constituyen factores importantes en el retardo del crecimiento observado en niños menores de cinco años de edad.

3. Diferentes e importantes eventos que ocurren antes de los 6 meses de vida. De todos ellos, el bajo peso al nacer (BPN) y la prematuridad son dos factores que figuran como resultado de las inadecuadas condiciones de salud que la madre experimenta durante el primer trimestre del embarazo. El bajo peso al nacer (BPN) es el principal factor debido a su fuerte asociación con un mayor riesgo de morbilidad y mortalidad por enfermedades infecciosas, con tendencia a mantener bajo peso y tener muy baja resistencia ante la presencia de enfermedades durante la niñez y la adultez. Asimismo, las mujeres que nacieron con bajo peso al nacer cuando alcancen la edad adulta y resulten embarazadas, tendrán hijos con mayor riesgo de presentar también bajo peso al nacer lo que reproduce de esta manera de generación en generación el retardo en el crecimiento. Respecto de la prematuridad de los elementos aquí priorizados solo hemos considerado la anemia como factor causal relevante en vista de las evidencias disponibles; sin embargo, se debe mencionar que no está esclarecido de manera definitiva el rol de los programas de asistencia alimentaria durante el embarazo. De otra parte, la adquisición de infecciones, tanto del tracto urinario como las enfermedades de transmisión sexual (ETS), también es causante del bajo peso al nacer. (MEF, 2008). 
Figurara 1

Indicadores de Resukado del Programa Articulado Nutricional

Resultados

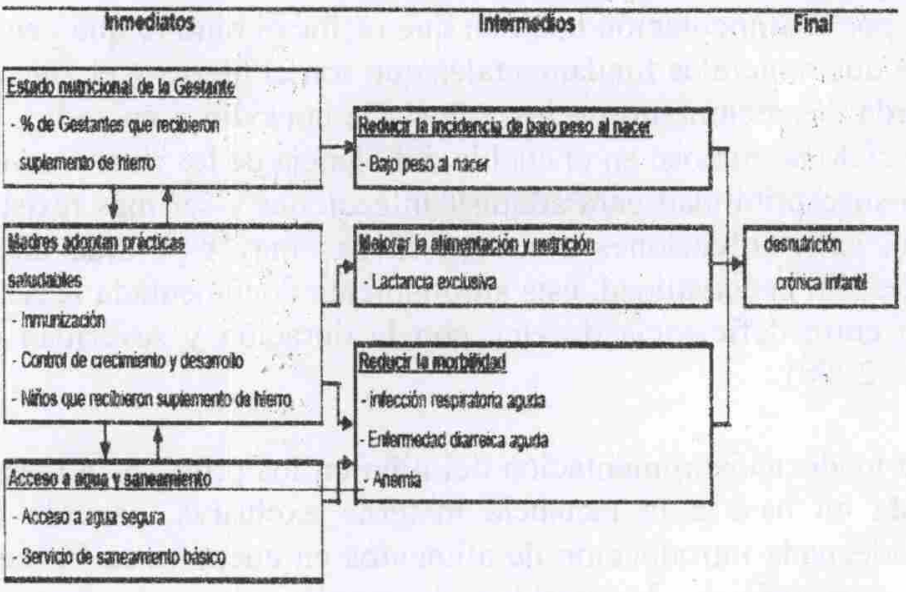

A manera de resumen, y de acuerdo con el modelo lógico, para lograr la reducción en la desnutrición crónica, es necesario: i) reducir la incidencia de bajo peso al nacer, ii) mejorar la alimentación y nutrición posterior del niño (36 meses) y iii) reducir la incidencia de enfermedades respiratorias y diarreicas (IRA y EDA respectivamente) y anemia del niño. A estas condiciones previas se les denominó resultados intermedios, pues conseguir estos cambios requería, a su vez, de otros resultados adicionales que se denominaron resultados inmediatos y que demandaban intervenciones concretas, denominadas productos, los cuales se focalizan en dos momentos del ciclo de vida del niño: durante la gestación y sus primeros años de vida. (MEF, 2008). De acuerdo con el modelo lógico las intervenciones (productos) del PAN llevan a resultados inmediatos, intermedios y final. La Dirección Nacional de Presupuesto Público del MEF, en el marco del Presupuesto por Resultados, suscribió dos Convenios con el Instituto Nacional de Estadística e Informática (INEI) para generar, entre otros, indicadores para realizar el seguimiento y medir los resultados del Programa Articulado Nutricional. En este contexto, el INEI ha diseñado un conjunto de indicadores denominados indicadores de resultado para el PAN, cuya relación se encuentra en la Figura l organizados según resultados inmediatos, intermedios y final (INEI, 2012). La propuesta de investigación utilizará los indicadores de resultado en referencia para estimar la magnitud de las desigualdades sociales correspondiente a cada intervención del PAN. 


\section{Aspectos metodológicos}

Este artículo utiliza como fuente de información los datos de las Encuestas Nacionales de Demografía y de Salud Familiar (ENDES) realizadas en los años 2007 y 2011 por el Instituto Nacional de Estadística e Informática (INEI) con el objetivo de proveer, entre otros, de información actualizada sobre la dinámica demográfica y el estado de salud de las madres y niños menores de cinco años que permita la estimación de los indicadores identificados en los Programas Estratégicos en el marco de la Estrategia Nacional de Presupuesto por Resultados. (ENDES, 2011). Las ENDES en mención son investigaciones por muestreo que proporcionan información sobre los hogares y la población objetivo constituida por las mujeres en edad reproductiva entre 15 y 49 años de edad y sobre los hijos tenidos en los cinco años anteriores a la entrevista. Conforme a los procedimientos muestrales las ENDES tienen representatividad a nivel nacional, departamentos, regiones naturales (Lima Metropolitana, resto de la Costa, Sierra y Selva) y área urbana y rural de residencia. Hemos utilizado información del cuestionario del hogar relacionado con características de los hogares y sus miembros (mujeres entre 15 y 45 años de edad y niños menores de cinco años); del cuestionario individual sobre antecedentes de la entrevistada, reproducción, embarazo, parto, lactancia, inmunización y salud; y del módulo de antropometría en cuanto a peso y talla.

Las intervenciones (productos) se han operacionalizado según la definición conceptual establecida por el Instituto Nacional de Estadística e Informática. En el Cuadro 1, se presentan los indicadores de resultado en referencia organizados según resultados inmediatos, intermedios y final del Programa Articulado Nutricional. La evidencia empírica reportada en la literatura

KUADRO 1

Perú: Intervenciones e Indicadores de Resultado del Programa Articulado Nutricional

\begin{tabular}{|c|c|c|}
\hline Resultados & Intervenciones & Indicadores de Resultado \\
\hline N & $\begin{array}{l}\text { Gestanites que recibrerosi } \\
\text { suplemento de hieno }\end{array}$ & 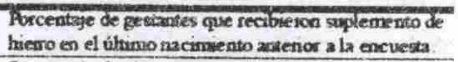 \\
\hline $\mathbf{M}$ & Inmumiacios & $\begin{array}{l}\text { Porcentsye de nibios menores de } 36 \text { me ses con vacunas } \\
\text { compleras para ga edad. }\end{array}$ \\
\hline $\begin{array}{l}0 \\
1\end{array}$ & Control de crecimiento y desarrollo & 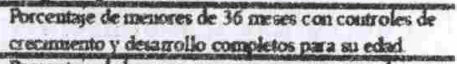 \\
\hline$\stackrel{A}{T}$ & Acceso a agua segata & Porcentaje de hegares can acceso a aguia trats da \\
\hline $\begin{array}{l}0 \\
\mathbf{S}\end{array}$ & Servicios de snneamiento básico & Porcentaje de hogares con sameamiento básico. \\
\hline I & Peso al nacer & 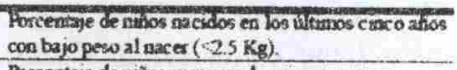 \\
\hline $\mathrm{r}$ & Lactancia & $\begin{array}{l}\text { Porcentaje de nitios menores de sess meses con } \\
\text { bctaocia materna exclusiva }\end{array}$ \\
\hline $\begin{array}{l}\mathbf{E} \\
\mathbf{R}\end{array}$ & $\begin{array}{l}\text { Natios que recibieron mplemento } \\
\text { de hiemo }\end{array}$ & $\begin{array}{l}\text { Pocerntaje de nidos de } 6 \text { a mesos de } 36 \text { meses gue } \\
\text { recibierca suplemento de hierro. }\end{array}$ \\
\hline$\frac{\text { II }}{\mathbf{E}}$ & Inieccios reqperateris a guda (IRA) & $\begin{array}{l}\text { Porcenteje de vidos menores de } 36 \text { meses que eal } \\
\text { bs dos semanas anteriotes a la encuesta tuvieron } \\
\text { infec ción respuratora aguda. }\end{array}$ \\
\hline \multirow[t]{2}{*}{$\begin{array}{l}1 \\
0 \\
5\end{array}$} & Enifemedad diarreica aguda (EDA) & $\begin{array}{l}\text { Porcentaje de numos menores de } 36 \text { meses que en } \\
\text { bs dos semonas antenores a la enciesta tuvieron } \\
\text { enfermedad diarreica aguda. }\end{array}$ \\
\hline & Anemis & $\begin{array}{l}\text { Porcentaje de ntilos extre } 6 \text { y } 35 \text { meses de extad con } \\
\text { anconia en las } 4 \text { aemusas antesiores a la excuesta. }\end{array}$ \\
\hline FTNAL & Desmuarición cróntica & $\begin{array}{l}\text { Porcentrye de niblob menores de cinco alas } \\
\text { con desnutrición csúnxa de acues do con } \\
\text { el patrón NCHS de seferencia. }\end{array}$ \\
\hline
\end{tabular}


revela que para medir las desigualdades pueden utilizarse diferentes medidas. Cada medida tiene sus ventajas y desventajas y sirve para diferentes propósitos. Por ello, y con la finalidad de tener una visión completa de las desigualdades sociales, es recomendable estimar y comparar varias medidas. La literatura en referencia muestra una fuerte preferencia por tres tipos de medidas: el cociente y diferencia entre grupos, el índice de concentración y el indice relativo de desigualdad. (Borrell et. al., 2000; Sandiford y Salvetto, 2000; Wafstaff et al, 1991; Manor et al, 1997). En consecuencia, para estimar la magnitud y analizar las tendencias de las desigualdades sociales existentes en cada una de las intervenciones del Programa Articulado Nutricional 2007-2011 organizadas según resultados inmediatos, intermedios y final se utilizará el cociente y diferencia entre grupos, el índice de concentración (IC) y el índice relativo de desigualdad (IRD).

El cociente y diferencia entre grupos son las medidas frecuentemente utilizadas en el estudio de las desigualdades sociales en salud. Básicamente, sirven para comparar el estado de salud entre dos grupos socioeconómicos. Generalmente, son medidas de efecto $\sin$ impacto total, ${ }^{4}$ a no ser que se comparen grupos extremos con igual número de individuos (por ejemplo, quintiles). Pueden no tener en cuenta todos los grupos, como por ejemplo al comparar el grupo I con el V, o pueden comparar dos grupos que constituyen toda la población; por ejemplo, trabajadores no manuales y trabajadores manuales. (Borrell et. al., 2000). Su ventaja principal es la flexibilidad cuando se trata de escoger entre los grupos que se quieren comparar. La elección de los grupos debe evitar que sean grupos tan extremos que oculten la información de los grupos intermedios o que sean grupos tan amplios que se diluyan las diferencias existentes. Otras ventajas a destacar son su facilidad de cálculo e interpretación. Entre las principales limitaciones destaca el hecho de que habitualmente no tienen en cuenta los grupos intermedios y, en este caso, las desigualdades serían las mismas si los grupos intermedios variaran y los grupos extremos se mantuvieran. Borrell, citando a Wagstaff et al., refiere que el hecho que estas medidas no tengan en cuenta el tamaño de los grupos que se comparan, a no ser que se elijan grupos de

${ }^{4}$ Medidas de efecto son medidas que tienen en cuenta el efecto que el nivel socioeconómico tiene en la salud. Contrariamente, las medidas de impacto total tienen en cuenta no solo el efecto que el nivel socioeconómico tiene en la salud, sino también el tamaño de los grupos afectados por las desigualdades (por ejemplo los grupos sociales) y por lo tanto, añaden información a las medidas de efecto. Son medidas útiles al estudiar las tendencias de las desigualdades sociales en salud en el caso que la distribución de la población en los grupos sociales haya cambiado. (Borrell et. al., 2000). 
igual tamaño (por ejemplo, los quintiles extremos), puede afectar las comparaciones temporales o comparaciones entre diferentes áreas geográficas si existen importantes diferencias temporales o espaciales en el tamaño de los grupos. (Borrell et. al., 2000).

En la propuesta de investigación, se estimará el cociente entre grupos para cada una de las intervenciones del Programa Estratégico Articulado, organizadas según resultados inmediatos, intermedios y final. Así, por ejemplo, si para el resultado final desnutrición crónica infantil el porcentaje de desnutrición crónica entre el quintil inferior y superior de bienestar es de 35.6 y $1.8 \%$ en el año 2011 , el cociente entre grupos será de $19.7^{5} \mathrm{El}$ cociente entre grupos indica que en el quintil inferior de bienestar hay 19.7 veces más niños con desnutrición crónica que en el quintil superior de bienestar. Ver Anexo.

A su vez, la diferencia entre grupos, como su nombre lo indica, es la diferencia entre el indicador del grupo comprendido en el quintil inferior y superior de bienestar. En la propuesta de investigación, se estimará la diferencia entre grupos para cada una de las intervenciones del Programa Estratégico Articulado organizadas según resultados inmediatos, intermedios y final. Para el ejemplo precedente, la diferencia entre los porcentajes de desnutrición crónica infantil entre los grupos comprendidos en el quintil inferior y superior de bienestar en el año 2011 es de 33.8 (35.6-1.8). El cociente entre grupos indica que la diferencia entre los porcentajes de desnutrición crónica infantil entre los quintiles inferior y superior es de $33.8 \%$. Ver Anexo.

Otra de las medidas ampliamente utilizada para representar la magnitud de la desigualdad es el coeficiente de Gini, propuesto por Gini en 1912, el cual se deriva de la curva de Lorenz. Se ha utilizado sobre todo para estudiar las desigualdades en la distribución del ingreso, aunque también se utiliza en el estudio de las desigualdades en salud. La curva de Lorenz se representa en el plano uniendo puntos $(x, y)$ que indican dos proporciones. En el eje de las $\mathrm{X}$, se representa la proporción acumulada de población ordenada según la salud y en el eje de las $Y$, la proporción acumulada de individuos afectados por la característica de estudio entre el total de la población de afectados. (Borrell et. al., 2000).

\footnotetext{
${ }^{5}$ Las ENDES 2007 y 2011 definen el nivel socioeconómico de los hogares en cinco quintiles de bienestar: quintil inferior, segundo quintil, quintil intermedio, cuarto quintil, y quintil superior. (ENDES, 2012).
} 
Si la salud se distribuye homogéneamente entre la población, la curva se sitúa en diagonal; en cambio, cuanto más se aleja la curva de la diagonal, mayor es el grado de desigualdad. La desigualdad es máxima cuando la curva de Lorenz forma un ángulo recto. El coeficiente de Gini se obtiene multiplicando por 2 el área entre la curva y la diagonal y por lo tanto, varía entre 0 cuando la igualdad es completa y 1 cuando la desigualdad es máxima (en este último caso el área entre la curva y la diagonal vale 0,5 , área de un triángulo rectángulo con base y altura igual a 1). Esto tiene la ventaja de reflejar la situación socioeconómica de todos los individuos. Entre sus limitaciones cabe destacar que para su estimación no siempre se dispone de datos de forma individual y es difícil el ajuste por edad y el cálculo de los intervalos de confianza. (Borrell et. al., 2000).

Si se ordena la población según el estatus socioeconómico, se consigue incluir la dimensión socioeconómica en la medición y generar una curva y un indice denominados curva de concentración e indice de concentración. La curva e índice de concentración se calculan con el mismo método que la curva de Lorenz y el coeficiente de Gini, pero incorporando la dimensión social. El índice de concentración toma valores entre -1 y +1 . Cuanto mayor es el valor del índice de concentración, mayor es la desigualdad. Un índice de concentración igual a 0 no indica ausencia de desigualdad (porque podría ser que la curva de concentración cruzara la diagonal y los valores positivo y negativo de las áreas se compensaran) sino ausencia de gradiente según grupos.

En la propuesta de investigación, se estimará el indice de concentración y graficará la curva de concentración para cada una de las intervenciones del Programa Estratégico Articulado, organizadas según resultados inmediatos, intermedios y final. Así, por ejemplo, para el resultado final desnutrición crónica infantil el indice de concentración es de -0.447 . El valor del índice de concentración implica una importante desigualdad y el valor negativo corresponde a una curva de concentración por encima de la diagonal, lo cual significa que los quintiles más pobres acumulan una cantidad niños con desnutrición crónica infantil superior a la que era de esperar. Ver Anexo.

\section{4.- Resultados}

En los cuadros 2 y 3 , se presentan los indicadores de resultados inmediatos, intermedios y final del Programa Articulado Nutricional organizados según quintiles de bienestar entre los años 2007 y 2011 expresados en porcentajes. En términos generales, los indicadores de resultado inmediatos muestran, por un lado, un aumento en los porcentajes promedio entre los años 2007 y 2011 principalmente en el indicador de resultado porcentaje de hogares rurales 
con servicios de saneamiento básico que aumentó de 59.3 para 71.1 por ciento; por otro, una relación bastante marcada con el nivel socioeconómico (expresado en términos de quintiles de bienestar) porque conforme aumenta el nivel socioeconómico aumentan los porcentajes correspondientes a los indicadores de resultados inmediatos; así, en el año 2007 los porcentajes de hogares rurales con servicios de saneamiento básico aumentaron de 41.1 por ciento en el quintil inferior para 100.0 por ciento en el quintil superior y en el año $2011 \mathrm{el}$ aumento fue de 63.9 por ciento en el quintil inferior para 100.0 por ciento en el quintil superior.

De forma general, el comportamiento de los indicadores de resultados inmediatos del Programa Articulado Nutricional muestra entre los años 2007 y 2011 un aumento importante en los porcentajes promedio relacionados principalmente con la cobertura de los servicios de agua segura y saneamiento básico especialmente en los hogares rurales. Este aumento en los porcentajes promedio está sustentado básicamente en un aumento importante en los porcentajes de cobertura de los servicios de agua y saneamiento básico en los hogares urbanos y principalmente en el saneamiento básico en los hogares rurales comprendidos en los quintiles I, II y III de bienestar.

Contrariamente, y conforme a lo esperado, los indicadores de resultado intermedios muestran, por un lado, una disminución en los porcentajes promedio, principalmente los indicadores de resultado porcentaje de niños menores de 36 meses con infección respiratoria aguda, con enfermedad diarreica aguda y con anemia, los cuales disminuyeron en $3.6,2.9$ y 15.0 puntos porcentuales entre los años 2007 y 2011 . Por otro, muestran también una importante relación con el nivel socioeconómico porque conforme aumenta el nivel socioeconómico disminuyen los porcentajes de niños con infección respiratoria aguda, con enfermedad diarreica aguda y con anemia. Así, el porcentaje de niños con infección respiratoria aguda disminuyó de 28.3 para 15.4 por ciento entre el quintil inferior y quintil superior de bienestar el año 2007 y de 20.8 para 12.6 por ciento entre los quintiles I y V de bienestar en el 2011. De la misma forma, el comportamiento del indicador de resultado final porcentaje de niños menores de cinco años con desnutrición crónica muestra una disminución de 22.9 para 15.2 por ciento entre los años 2007 y 2011 y una relación con el nivel socioeconómico porque conforme aumenta el nivel socioeconómico de los niños menores de cinco años de edad disminuye significativamente los porcentajes de desnutrición crónica infantil de 47.5 y 35.6 por ciento en el quintil inferior para 4.7 y 1.8 por ciento en el quintil superior entre los años 2007 y 2011 , respectivamente 
Cuadro 3

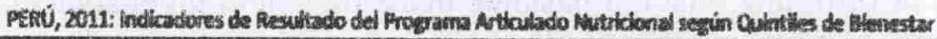

\begin{tabular}{|c|c|c|c|c|c|c|}
\hline \multirow[b]{2}{*}{ Indicadores do lessultado } & \multicolumn{5}{|c|}{ Quintiles de Bienestax } & \multirow[b]{2}{*}{ Promedo } \\
\hline & 8 (thierion) & II & 情 & IV & V (sugerion) & \\
\hline \multicolumn{7}{|l|}{ 1. Resultados imediaces } \\
\hline \% de gestantes que redibieron suplemento de hierro & 84.4 & 85.5 & 87.0 & 91.6 & 92.4 & 87.8 \\
\hline \% de hogares con acreso a aqua sequra & 79.5 & 91.2 & 97.4 & 99.5 & 99.9 & 93.3 \\
\hline W de hogares con servicios de ssmeamienta bassico & 63.5 & 82.4 & 94.7 & 99.2 & 100.0 & 87.6 \\
\hline \% de hogares rurates con servicios de sancaniento hásico & 63.9 & 79.9 & 86.4 & 92.6 & 100.0 & 71.1 \\
\hline \multicolumn{7}{|l|}{ 2.- Resutados intermedios } \\
\hline \% de niftos con bajo peso al nacer & 9.4 & 8.1 & 6.5 & 5.5 & 5.2 & 7.1 \\
\hline \% dc nifías a de 6 meses con lactancia materna excliesiva & 85.3 & 79.5 & 66.3 & 66.7 & 34.6 & 70.6 \\
\hline Ho de nifus 436 meses con intecciòn respiratoria aqudh & 20.8 & 18.3 & 19.1 & 15.5 & 12.9 & 17.8 \\
\hline \% de niños e 36 meses con entermedad diarreics aguda & 20.3 & 20.4 & 18.8 & 15.9 & 89 & 17.7 \\
\hline \% de nilhos eritre 6635 meses con anemia & 49.7 & 49.3 & 41.1 & 33.8 & 24.1 & 41.6 \\
\hline \multicolumn{7}{|l|}{ 3. Resultase Find } \\
\hline \% de niflos e 5 años con desurtrición crónics infantil & 35.6 & 17.1 & 7.4 & 4.0 & 1.8 & 15.2 \\
\hline
\end{tabular}

Cusche?

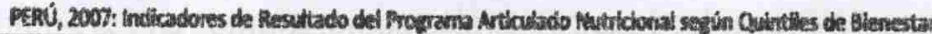

\begin{tabular}{|c|c|c|c|c|c|c|}
\hline \multirow[b]{2}{*}{ Indicoviares de lesaltado } & \multicolumn{5}{|c|}{ Quintiles de Bienestar } & \multirow[b]{2}{*}{ Promedilo } \\
\hline & I (niferion) & H & 提 & N & $V$ (Superion) & \\
\hline \multicolumn{7}{|l|}{ 1. Resultados inanedatos } \\
\hline \% de gestantes que recibieron suphemento dierfo & 71.5 & 74.7 & 73.5 & 81.0 & 82.9 & $\pi 6.6$ \\
\hline \% de hogares con acceso a agua segura & 68.2 & 87.9 & 95.9 & 99.2 & 99.9 & 92.) \\
\hline \% de hogares con scrvicios de santamiento basico & 41.6 & 62.4 & 86.6 & 98.1 & 100.0 & 816 \\
\hline *o de hogares furales con servicios de saneamiefto básico & 41.1 & 62.3 & 79.9 & 91.2 & 100.9 & $\$ 9.3$ \\
\hline \multicolumn{7}{|l|}{ 2. Resuleados intemedilos } \\
\hline \% de nifics con bajo peso al nacer & 10.8 & 8.4 & 6.0 & 9.8 & 4.9 & 7.7 \\
\hline H de niflos \& da 6 meses con lactancia materna exciusira & 79.6 & 73.5 & 68.3 & 58.2 & 45.3 & 65.4 \\
\hline \% de aiflos e. 36 meses con infection respir atoria aguds & 28.3 & 22.9 & 20.7 & 20.0 & 15.4 & 21.4 \\
\hline \% de nisios $\times 36$ meses con eriemedad diareica aguda & 24.0 & 21.5 & 23.7 & 17.2 & 14.2 & 20.4 \\
\hline Ho de nifhos entre 6635 meses con anemia & 62.8 & 61.1 & 59.0 & 50.8 & 44.3 & 56.6 \\
\hline \multicolumn{7}{|l|}{ 3. Resulado finat } \\
\hline \%o de nifios e 5 zios con desnutrición crónica infantil & 47.5 & 33.4 & 16.8 & 8.3 & 4.7 & 22.9 \\
\hline
\end{tabular}

Fuente: INE1. ENOES 2U67. Elaboración or oripis.

Si bien los porcentajes promedio correspondiente a los indicadores de resultado inmediatos, intermedios y final muestran una tendencia en el comportamiento conforme lo esperado entre los años 2007 y 2011 ; sin embargo, es de relevar que las tendencias en el ritmo de aumento o decremento entre los indicadores de resultado en referencia han sido bastante diferenciados según el nivel socioeconómico. Así, y a manera de ejemplo, en el quintil I de bienestar los porcentajes entre los indicadores de resultado 
inmediatos varían entre 41.1 y 71.5 por ciento en el año 2007 para 82.9 y 100 por ciento en el quintil $\mathrm{V}$ de bienestar en el año 2011. De la misma forma, los porcentajes para los indicadores de resultado intermedio variaban entre 10.8 y 79.6 por ciento en el quintil inferior para 4.9 y 45.3 por ciento en el quintil $V$ de bienestar en el año 2007. Luego de cinco años de ejecución del Programa Articulado Nutricional, los porcentajes entre los indicadores de resultado inmediatos variaban entre 63.5 y 84.4 por ciento para el quintil I de bienestar y entre 92.4 y 100.0 por ciento para el quintil V de bienestar en el 2007 lo que revela una importante mejoría en las condiciones de vida en los hogares, principalmente los comprendidos en los quintiles I, II y III de bienestar.

Estas diferencias en las tendencias en el comportamiento de los indicadores de resultados del Programa Articulado Nutricional según quintiles de bienestar, tanto para el año 2007 cuanto para el 2011, se expresan en un aumento o disminución de las brechas existentes en cada uno de los indicadores de resultados según el nivel socioeconómico - medido en términos de quintiles de bienestar- $\mathrm{y}$ en la literatura sobre las desigualdades sociales, expresan desigualdades a favor de ciertos grupos con relación a determinados grupos sociales, las cuales pueden medirse utilizando medidas de desigualdad como el cociente y diferencia entre grupos y el índice de concentración.

En los cuadros 4 y 5 se puede observar el cociente (Q1/QV) entre grupos, la diferencia entre grupos (Q1-QV) y el indice de concentración (IC) para cada uno de los indicadores de resultado inmediatos intermedios y final del

Cuadro 4

Perú 2007: Cociente y Diferencia entre Grupos e findice de Concentración según indicadores de Resultado

\begin{tabular}{|c|c|c|c|}
\hline Indicadores de Resultado & $Q 1 / Q V$ & Q1-QV & K. \\
\hline $\begin{array}{l}\text { 1. - Resultados Inmedlatos } \\
\text { - \% de gestantes que recibieron suplemento de hierro } \\
\text { - \% de hogares con acceso a aqua segura } \\
\text { - \% de hogares con servicios de saneamiento básico } \\
\text { - \% de hogares rurales con servicios de saneamiento básico } \\
\text { 2.- Resultados intermedios } \\
\text { - \% de nihos con bajo peso al nacer } \\
\text { - \% de nihos < de } 6 \text { meses con lactancia materna exciusiva } \\
\text { - \% de niగhos < } 36 \text { meses con infección respiratoria aguda } \\
\text { - \% de nihos < } 36 \text { meses con enfermedad diarreica aguda } \\
\text { - \% de niños entre } 66-35 \text { meses con anemia } \\
\text { 3.- Resultado Final } \\
\text { - \% de nifos < } 5 \text { aflos con desnutrición crónica infantil }\end{array}$ & $\begin{array}{l}2.20 \\
1.76 \\
1.84 \\
1.69 \\
1.42\end{array}$ & $\begin{array}{r}5.90 \\
38.30 \\
12.90 \\
9.80 \\
18.50 \\
42.80\end{array}$ & $\begin{array}{l}-0.075 \\
-0.096 \\
-0.091 \\
-0.093 \\
-0.058 \\
-0.359\end{array}$ \\
\hline
\end{tabular}


Programa Articulado Nutricional para los años 2007 y 2011.

De acuerdo con las estimaciones presentadas en los cuadros 4 y 5 , los cocientes entre grupos para las intervenciones acceso a agua segura y servicios de saneamiento básico han aumentado entre los años 2007 y 2011 de 0.7 y 0.4 para 0.8 y 0.7 , respectivamente, lo que demuestra una pequeña disminución de las desigualdades sociales entre los hogares comprendidos entre los quintiles I y V de bienestar.

En cuanto a los indicadores de resultados intermedios, los cocientes entre grupos estimados revelan que en el quintil I inferior de bienestar hay en el año 2007 entre 1.4 y 2.2 veces y en el año 2011 entre 1.7 y 2.5 veces más niños menores de cinco años con bajo peso al nacer, infección respiratoria aguda, enfermedad diarreica aguda o anemia comparados con el quintil $\mathrm{V}$ de bienestar. A nivel de cada indicador de resultado, observamos, por un lado, que el cociente entre grupos para los indicadores de resultado porcentaje de niños con bajo peso al nacer y porcentaje de niños con infección respiratoria aguda ha disminuido de 2.2 para 1.9 y de 1.9 para 1.6 veces entre 2007 y 2011 , lo cual es indicativo de una disminución de las desigualdades sociales entre los grupos sociales de niños comprendidos entre los quintiles inferior y superior de bienestar.

Contrariamente, es de revelar que paralelamente entre los años 2007 y 2011 han aumentado los cocientes entre grupos para los indicadores de resultado porcentaje de niños con lactancia materna exclusiva, porcentaje de niños con enfermedad diarreica aguda y porcentaje de niños con anemia de 1.8 para 2.5 veces; de 1.7 para 2.3 veces y de 1.4 para 2.0 veces, respectivamente, lo que manifiesta un aumento en las desigualdades sociales entre los dos grupos extremos de bienestar.

Respecto del indicador de resultado final, los cocientes entre grupos estimados para los años 2007 y 2011 revelan que en el quintil I de bienestar existían 10.1 y 19.8 veces más niños menores de cinco años con desnutrición crónica infantil que en el quintil V de bienestar; además, revelan que estas desigualdades sociales se incrementaron entre el 2007 y 2011 
El análisis de las desigualdades sociales utilizando el indicador diferencia entre grupos muestra importantes diferencias entre los indicadores de resultado inmediatos. Así, en el año 2007, las diferencias entre los quintiles I y $\mathrm{V}$ de bienestar para los indicadores de resultado porcentaje de hogares con acceso a agua segura y porcentaje de hogares con servicios de saneamiento básico eran de 31.7 y 58.9 puntos porcentuales. Para el año 2011, si bien estas diferencias han disminuido sustantivamente, persisten diferencias porcentuales de 20.4 y 36.4 puntos entre los indicadores de resultado en análisis. Estos resultados revelan, de forma general, la persistencia de diferencias sociales en favor de los hogares comprendidos en los quintiles de mayor bienestar.

Cuadro 5

Perú 2011: Cociente y Dfferencia entre Grupos e indice de Concentración según indicadores de Resultado

\begin{tabular}{|c|c|c|c|}
\hline Indicadores de Resultado & Q1/QV & Q1-QV & Valor \\
\hline \multicolumn{4}{|l|}{ 1.- Resultados Inmediatos } \\
\hline \% de gestantes que recibieron suplemento de hierro & 0.91 & 8.00 & 0.018 \\
\hline -\% de hogares con acceso a agua segura & 0.80 & 20.40 & 0.043 \\
\hline - \% de hogares con servicios de saneamiento básico & 0.64 & 36.40 & 0.082 \\
\hline -\% de hogares rurales con servicios de saneamiento básico & 0.68 & 30.50 & 0.064 \\
\hline \multicolumn{4}{|l|}{ 2.- Resulkados intermedios } \\
\hline -\% de niños con bajo peso al nacer & 1.81 & 4.20 & -0.121 \\
\hline - \% de nifios $<$ de 6 meses con lactancia materna exclusiva & 2.47 & 50.70 & -0.100 \\
\hline - \% de niños < 36 meses con infección respiratoria aguda. & 1.65 & 8.20 & -0.076 \\
\hline - \% de niños < 36 meses con enfermedad diarreica aguda & 2.28 & 11.40 & -0.102 \\
\hline - \% de niños entre $66-35$ meses con anemia & 2.06 & 25.60 & -0.113 \\
\hline \multicolumn{4}{|l|}{ 3.- Resulkado Final } \\
\hline - \% de niños < 5 años con desnutrición crónica infantil & 19.78 & $33.800^{6}$ & -0.447 \\
\hline
\end{tabular}

Fuente: INEI. ENDES 2011. Elaboración prợia.

Con relación a los indicadores de resultado intermedios, las diferencias entre grupos estimadas revelan por un lado, una disminución en las diferencias entre los porcentajes correspondientes a los indicadores de resultado peso al nacer e infección respiratoria aguda de 5.9 para 4.2 y de 12.9 para 8.2 puntos porcentuales entre los años 2007 y 2011. De otro lado, un aumento en las diferencias en referencia entre los indicadores de resultado lactancia materna exclusiva, enfermedad diarreica aguda y anemia, que aumentan de 34.3 para 50.7 , de 9.8 para 11.4 y de 18.5 para 25.6 puntos porcentuales respectivamente. En ambos casos, la disminución o aumento en las diferencias se debe principalmente a la mayor disminución relativa en los 
porcentajes correspondiente a niños ubicados en los quintiles IV y V de bienestar y no necesariamente en los quintiles I y II de bienestar, conforme lo esperado.

Para el caso del indicador de resultado final desnutrición crónica infantil, las diferencias entre grupos (quintil I y quintil V) muestran tanto para el año 2007 como para el 2011 importantes diferencias entre los quintiles extremos de bienestar (42.8 y 33.8 puntos porcentuales); paralelamente muestran también una importante disminución en las diferencias entre los quintiles extremos mencionados de 9.0 puntos porcentuales, debido principalmente a una importante disminución en los porcentajes de niños con desnutrición crónica infantil comprendidos en los quintiles I y II de bienestar.

Utilizando el índice de concentración como una medida de desigualdad observamos bajos y positivos índices de concentración para los indicadores de resultado inmediatos tanto para el año 2007 como para el 2011. Estos resultados revelan pequeñas desigualdades sociales en favor de los grupos sociales comprendidos en los quintiles de mayor bienestar relacionado con características de los hogares en cuanto a acceso a agua segura y saneamiento básico. La misma información revela también que estas pequeñas desigualdades sociales han disminuido entre los años 2007 y 2011.

Contrariamente, el valor negativo de los índices de concentración estimados para los indicadores de resultado intermedios entre los años 2007 y 2011, revelan por un lado, importantes desigualdades sociales en contra de los grupos sociales de menor bienestar, principalmente los relacionados con los indicadores de resultado peso al nacer y anemia; por otro, que estas desigualdades sociales se han incrementado entre el 2007 y 2011. En tanto que para el indicador de resultado final relacionado con la desnutrición crónica infantil en menores de cinco años, los índices de concentración estimados de -0.359 y -0.447 entre los años 2007 y 2011 , respectivamente, revelan por un lado, importantes desigualdades sociales en la desnutrición crónica infantil entre los grupos sociales y por otro, que estas desigualdades sociales se han incrementado significativamente entre los años 2007 y 2011, respectivamente.

\section{5.- Conclusiones y recomendaciones}

La implementación del Presupuesto por Resultados (PpR) y su ejecución a través de la puesta en operación de un conjunto de Programas Estratégicos como el Programa Articulado Nutricional (PAN) constituye una importante experiencia adquirida por el Ministerio de Economía y Finanzas en la Gestión por Resultados dirigida al diseño de un conjunto articulado de 
intervenciones y orientada hacia un resultado final. El modelo lógico del PAN establece un conjunto de intervenciones inmediatas e intermedias interrelacionadas y relacionadas con el resultado final desnutrición crónica infantil.

Como resultado de la implementación de las intervenciones comprendidas en el Programa Articulado Nutricional, los indicadores de resultado muestran importantes avances en el aumento disminución en los porcentajes relacionados con cada uno de los indicadores de resultado del Programa Articulado Nutricional. Debe resaltarse en el aumento en los porcentajes correspondientes a los indicadores de resultado inmediatos y disminución de los porcentajes correspondientes a los indicadores de resultado intermedios. En ambos casos, se observa una relación entre los indicadores de resultado y el nivel socioeconómico, expresado en términos de quintiles de bienestar. Los cocientes entre grupos para los años 2007 y 2011 evidencian por un lado, desigualdades sociales en contra de los grupos sociales comprendidos en los quintiles de menor bienestar; por otro, que estas desigualdades sociales han disminuido entre los años 2007 y 2011. De la misma forma, las diferencias entre grupos evidencia para algunos indicadores de resultado un aumento en las diferencias porcentuales relacionadas principalmente con indicadores de resultado intermedios y entre el 2007 y 2011 estas diferencias han aumentado para algunos indicadores y disminuido para otros. Para el indicador de resultado final, las diferencias entre grupos han aumento debido principalmente a una importante disminución de la desnutrición crónica infantil entre los niños comprendidos en los quintiles I y $\mathrm{V}$ de mayor bienestar.

Los índices de Gini estimados para los indicadores de resultado inmediatos e intermedios revelan de forma general leves desigualdades en contra de los grupos comprendidos en los quintiles inferiores de bienestar y que estas diferencias han tendido a disminuir entre los años 2007 y 2011. Pero para el indicador de resultado final, los índices de Gini estimados revelan importantes desigualdades sociales en contra de los grupos sociales comprendidos en los quintiles de menor bienestar y que estas desigualdades han mostrado una tendencia a incrementarse en el período 2007 y 2011.

El análisis de los indicadores de resultados del Programa Articulado Nutricional con la utilización de medidas de desigualdad permite exponer las siguientes recomendaciones: El Programa Articulado Nutricional de los próximos años debe prioritariamente orientarse a incrementar la cobertura con el objetivo de aumentar los porcentajes de hogares con servicios de agua segura y servicios de saneamiento básico, sobre todo en el área rural. Si bien 
resulta importante disminuir los porcentajes también resulta importante continuar disminuyendo las desigualdades sociales existentes, principalmente, entre los quintiles inferior y superior de bienestar.

De la misma forma, debe orientase a disminuir los porcentajes de niños con bajo peso al nacer, infección respiratoria aguda, enfermedad diarreica aguda y anemia, principalmente entre los niños comprendidos entre los quintiles I y II de menor bienestar con la finalidad de disminuir las desigualdades sociales que aún persisten y que incluso algunas, aumentaron entre los años 2007 y 2011 .

En cuanto al resultado final, el Programa Articulado Nutricional debe tener como objetivo final continuar con la disminución de los porcentajes de desnutrición crónica infantil principalmente entre los niños comprendidos entre los quintiles I y II de menor bienestar y debe buscar disminuir las desigualdades sociales existentes principalmente las relacionadas con resultados intermedios y final, algunos de las cuales se han incrementado de manera importante entre los años 2007 y 2011.

En coherencia con el resultado precedente, resulta necesario orientar las intervenciones que llevan a resultados inmediatos e intermedios del Programa Articulado Nutricional con el objetivo de disminuir las desigualdades existentes principalmente la relacionada con el resultado final desnutrición crónica infantil en niños menores de cinco años. La utilización de medidas de desigualdad social en nuestro país se ha llevado a cabo de manera esporádica y es de utilización reciente. En este contexto, y con la finalidad de evaluar los resultados obtenidos en el presente artículo, resulta pertinente evaluar las diferencias sociales utilizando otras medidas de desigualdad social. 


\section{ANEXOS}

ENDES 2011: DESNUTRICION CRONICA INFANTHL COEFICIEPATE Y DIFERENCIA ENTRE GRUPOS

\begin{tabular}{|c|c|c|c|c|}
\hline $\begin{array}{l}\text { Quintiles do } \\
\text { Bienestar }\end{array}$ & \multicolumn{2}{|c|}{ Total Miños $=5$ afios } & $\begin{array}{l}\text { Niños } \\
\text { con DC }\end{array}$ & $\begin{array}{r}\% \text { de Desnutrición } \\
\text { Crónica }\end{array}$ \\
\hline Quintil inferior & 2.177 & 24.7 & 774 & 35.6 \\
\hline 3egundo quintis & 2,012 & 22.8 & 344 & 17.1 \\
\hline Quintil intermedio & 1,962 & 22.2 & 145 & 7.4 \\
\hline Cuarto quintil & 1,562 & 17.7 & 62 & 4.0 \\
\hline Ountit superior & 1,111 & 12.6 & 20 & 1.6 \\
\hline Total & 8824 & 100.0 & 1345 & 15.2 \\
\hline $\begin{array}{l}\text { Cociente } \\
\text { Diferencia entre gr }\end{array}$ & & & & $\begin{array}{l}19.7 \\
33.8\end{array}$ \\
\hline
\end{tabular}

\section{Figura $\mathbf{N}^{\circ} 1$}

\section{CURVAE IUDDICE DE CONCENTRACIÓN}

\begin{tabular}{|c|c|c|c|c|c|c|c|}
\hline $\begin{array}{l}\text { Quintiles de } \\
\text { Bienestar }\end{array}$ & Niflos & Proporción & $\begin{array}{r}\text { Proporcion } \\
\text { Acumulada } \\
\text { (eje X) }\end{array}$ & $\begin{array}{r}\text { Niños } \\
\text { con DC } \\
\end{array}$ & Proporción & $\begin{array}{r}\text { Proporción } \\
\text { Acumulada } \\
\text { (eje Y) }\end{array}$ & $\begin{array}{c}\text { hodice de } \\
\text { Concentración }\end{array}$ \\
\hline Quntil inferior & 2177 & 0.247 & 0.247 & 774 & 0.575 & 0.575 & 0.142 \\
\hline Segundo quintil & 2012 & 0.228 & 0.475 & 344 & 0.256 & 0.831 & 0.321 \\
\hline Ouintil intermadio & 1962 & 0.222 & 0.697 & 145 & 0.108 & 0.939 & 0.394 \\
\hline Cuarto quintil & 1562 & 0.177 & 0.874 & 62 & 0.046 & 0.925 & 0.341 \\
\hline Quntil superior & 1111 & 0.126 & 1.000 & 20 & 0.015 & 1.000 & 0.250 \\
\hline Total & 8224 & 1.000 & 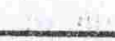 & 1345 & 1.000 & & 1.447 \\
\hline $\begin{array}{l}\text { Curva de consentrs } \\
\text { indice de concentr }\end{array}$ & Figur & & & & & & -0.447 \\
\hline
\end{tabular}

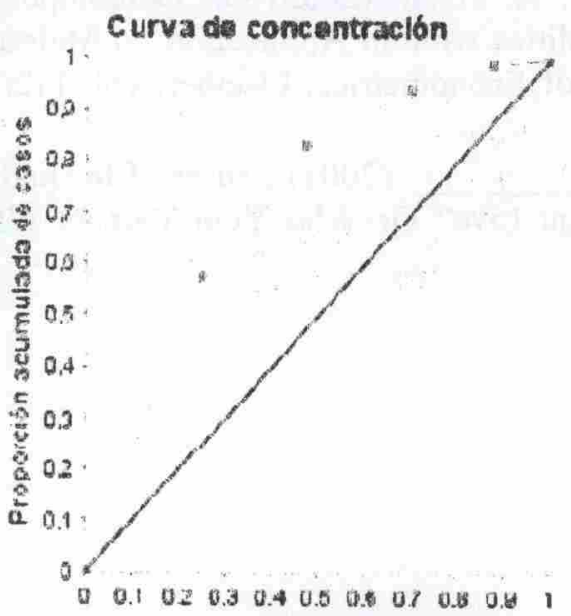

Ppoporcion acumulada de potascion 


\section{BIBLIOGRAFIA}

1. BORRELL et. al. (2000) La medición de las desigualdades en salud. Gaceta Sanitaria 2000;14 (Suplemento 3):20-33.

2. INSTITUTO NACIONAL DE ESTADÍSTICA E INFORMÁTICA (INEI). (2012) Perú: Encuesta Demográfica y de Salud Familiar 2011. Informe General

3.

(2012) Perú: Indicadores de

Resultados de los Programas Estratégicos, 2000-2011.

4. MAYSTON, D. (1999). The Economic Determinants of Health Inequalities" Department of Economics and Related Studies. University of York.

5. MINISTERIO DE ECONOMIA Y FINANZAS. (2008) Programa Articulado Nutricional.

6. ORGANIZACIÓN PANAMERICANA DE LA SALUD / Organización Mundial de la Salud

7. (OPS/OMS) (1999). Principios y Conceptos Básicos de Equidad y Salud. Washington, DC: Organización Panamericana de la Salud / Organización Mundial de la Salud (OPS/OMS).

8. SCHEIDER et., al., (20002) Métodos de medición de las desigualdades. Revista Panamericana de Salud Publica/Pan Am J Public Health 12(6).

9. SEGURA, J. L. et all. (2002) Pobreza y desnutrición infantil PRISMA: Lima, septiembre.

10. UNICEF (1998) Estado Mundial de la Infancia 1998. Fondo de las Naciones Unidas para la Infancia.

11. WASTGAFF, A. et all. (2003) On Descomposing the Causes of Health Sector Inequalities with an Application on Malnutrition Inequalities in Vietnam. Journal of Econometrics, Elsevier, vol. 112(1), págs. 207-223, january.

12.

(2001) Causes of Inequality in Health: Who

You Are? Where You Live? Or Who Your Parents Where?. Manuscrito, noviembre. 

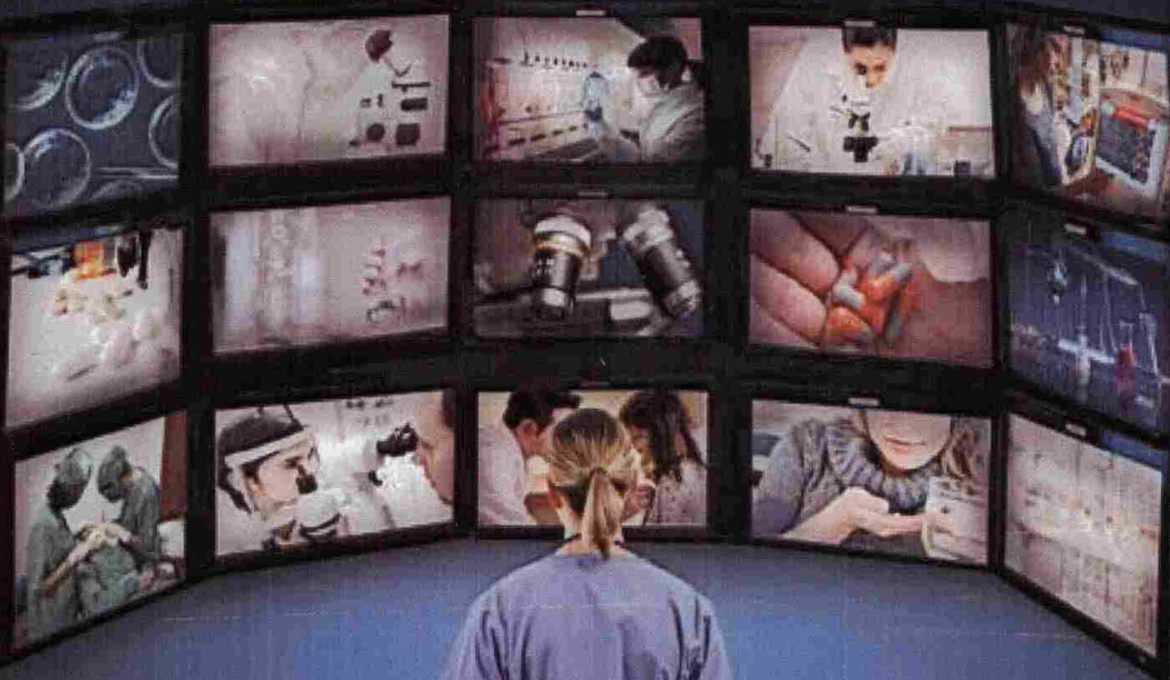

$\rightarrow$
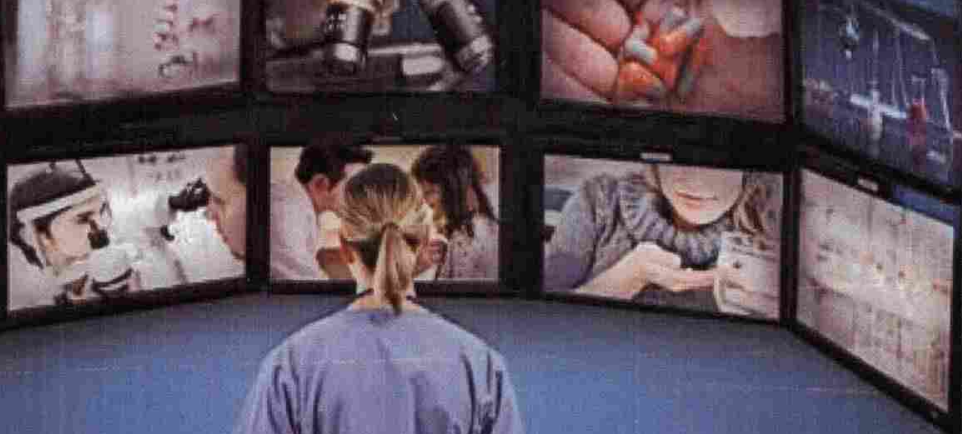

MONITOREA

\section{COORDINA Y SUPERVISA}

\section{Diplomado en Investigación Clínica para Coordinadores y Monitores de Estudios}

\section{OBJETIVO:}

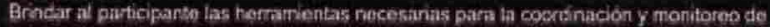
calidat de os estudias el inices, indepandienternenta dende se desarrollen

Inscripciones: hasta el 15 de marzo 2013

Entrevistas: 18 de marzo 2013

Inicio de clases: 18 de abril 2013

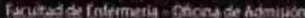

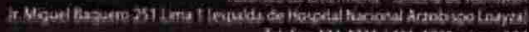

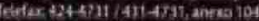

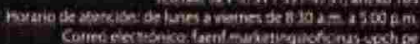

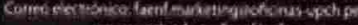

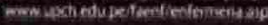


